\title{
MULTIDIMENSIONALITY IN POPULATION ANALYSIS
}

Nathan Keyfitz

RR-80-33

August 1980

Reprinted from Sociological Methodology 1980

INTERNATIONAL INSTITUTE FOR APPLIED SYSTEMS ANALYSIS

Laxenburg, Austria 
Research Reports, which record research conducted at IIASA, are independently reviewed before publication. However, the views and opinions they express are not necessarily those of the Institute or the National Member Organizations that support it.

Reprinted with permission from Sociological Methodology 1980, Jossey-Bass Publishers, San Francisco, 1979.

Copyright @ 1979 Jossey-Bass Publishers.

All rights reserved. No part of this publication may be reproduced or transmitted in any form or by any means, electronic or mechanical, including photocopy, recording, or any information storage or retrieval system, without permission in writing from the copyright holder. 


\section{FOREWORD}

Interest in human settlement and systems policies has been a central part of urban-related work at the International Institute for Applied Systems Analysis (IIASA) from the outset. From 1975 through 1978 this interest was manifested in the work of the Migration and Settlement Task, which was formally concluded in November 1978. Since then, attention has turned to disseminating the Task's results, to concluding its comparative study, and to exploring possible future work that might apply the newly developed mathematical methodology to other research topics.

During his visit to IIASA in 1978, Professor Nathan Keyfitz reviewed the work of the Migration and Settlement group carefully and explored with them possible new directions. This paper is the ultimate result of that review and exploration. The topics it describes are currently being examined by a number of scholars in the Human Settlements and Services Area, and it is likely that a new research task reflecting these interests in multidimensional or multistate demography will soon evolve.

Selected papers summarizing previous work on migration and settlement at IIASA are listed at the back of this paper.

ANDREI ROGERS

Chairman

Human Settlements and Services Area 


\section{PREFACE}

The paper that follows is an attempt to synthesize material from a number of sources, published originally under such headings as multi-regional demography, increment-decrement life tables, marriage tables, and tables of working life. In the elaboration of the several lines of work that are here put together, IIASA has had an important role. Through the activities of Andrei Rogers and his students and colleagues, a new discipline has come into existence that, on the one hand, unifies preceding work, and, at the same time, reaches out to new kinds of interdisciplinary work. The techniques that are sketched here not only embrace much of demography, but potentially they permit more convenient study of problems that require the union of demography and economics, demography and sociology, and other combinations of disciplines.

Demography, even more explicitly than other parts of social science, deals with transitions. People move from one age group to the next, from single to married, from labor force to retired, from living to dead. Of all the transitions that might be considered, the one between regions, i.e., migration, is the most general. Movement can (and usually does) take place between any pair of regions in any calendar period, while between ages, say, there is a restriction: if one survives one can only move from one age to the next higher age. Andrei Rogers started out to study migration, and his thorough exploration in this field led him and his students naturally to tables of mortality, of working life, of marriage and remarriage.

It is to be hoped that the following brief introduction to the field, written at IIASA, will help the reader see its main outlines, and encourage further reading. More detailed accounts are contained in the several Research Reports of IIASA referred to within this paper.

NATHAN Keyfitz 


\title{
MULTIDIMENSIONALITY IN POPULATION ANALYSIS
}

\author{
Nathan Keyfitz \\ HARVARD UNIVERSITY
}

Much of sociology and practically all of demography deals with transitions of people from one state at a certain moment to another state a year or 5 years later: single to married, married with one child to married with two children, at school to in the labor force, living to dead. These transitions have to be calculated from raw statistical data of various forms. Sometimes individual movements are registered: Robert Jones died on 23 April 1978; Mary Henderson gave birth to a baby boy on 17 July 1978; Henry

I am grateful to Jan Hoem, Pavel Kitsul, S. Krishnamoorthy, Jacques Ledent, Gary Littman, Frank Oechsli, David K. Pickard, Andrei Rogers, Robert Schoen, Mike Stoto, and Frans Willekens for opportunities to discuss the matters here presented. Frans Willekens, Andrei Rogers, and others corrected errors in earlier drafts. 
Johnson retired at the end of September 1978. The individual movements are aggregated into groups and published as official statistics: There were 1,372 deaths of males aged 50-54 in 1978; 987 girl babies were born to women aged 25-29 in 1976. Sometimes the data are not events but a count of the individuals in a region: 116,572 males aged $50-54$ were living in a certain area on 1 July 1978.

Before the sociology of the issues can be discussed-What are the class differences in mortality? Do working wives have fewer children than wives who stay home? - we have to translate the raw data of individual movements into probabilities of transition between points of time separated by 1 year, 5 years, or another suitable period. The model that makes mortality intelligible contains such fractions as the probability of a person alive at age 50 being dead at age 55 (or dead of a particular cause at age 55). Such a transition model corresponds to what would actually happen in a stationary population (same number of births year after year) in which the observed death rates prevail over a long period of time. It is called a life table.

The life table is a transition model in which observed death rates are the basis of probabilities of dying and then of the stationary population, the expectation of life, and other parameters of interest.

Migration analysis, on the other hand, often starts from a census question asking respondents where they were living 5 years earlier. A kind of transition probability is directly given by the aggregation of the resulting answers. With some qualifications one can thus in a sense observe transitions and infer moves from them; the opposite applies in mortality statistics, where it is moves that are observed and transitions inferred from them. That the Jones family lived in Denver in 1970 and in Omaha in 1975 is a transition; the family moved from Denver to Chicago in June 1971 and from Chicago to Omaha in November 1974.

Transitions are useful to know in many other fields. That there were just over 1 million divorces in the United States in 1977 tells a very small part of the story of marriage dissolution; anyone studying divorce wants to know the probability of divorce, say 
within 5 years of marriage, for couples in various categories. Only in that way can proper comparisons be made over time and between social groups. The original counts of numbers of divorces year by year do not even tell whether the propensity to divorce is increasing, let alone by how much.

That there were so many pupils in American schools, grade by grade, is again rather limited information. One wants to know the probability that a child starting school will go as far as seventh grade, eighth grade, ninth grade, and so on. Such probabilities or their summary in the expected length of schooling measure education at least quantitatively and are useful for comparing successive generations, income groups, and other segments of the population. The observed distribution by grades is too much affected by accidents of age distribution to be directly interpretable.

A couple is provided with some means of birth controlperhaps the wife is fitted with an IUD. What is the chance that the IUD will still be in place 1 month, 2 months, 3 months later? And how does this compare with the steadfastness of another couple in using a stock of pills with which they are provided? What is the chance that a recruit to a particular job will still be holding on and doing the work 1 year, 2 years, 20 years later?

All the hazards implied above-divorce, dropping out of school, failing to retain an IUD, losing a job-can be represented as hurdles at various heights proportional to the risk. When we know the runners who fail on the $i$ th hurdle as a fraction of the number who arrive at it, for all values of $i$, then the cumulative product of the probabilities of not failing tells us what fraction of the number that started the race will still be in the running after the $i$ th hurdle. This cumulative fraction remaining in the race after the $i$ th hurdle among those that started is the $l_{x}$ column of the ordinary singledecrement life table. The average number of hurdles cleared before the runner drops out is the expectation or $\dot{e}_{0}$ column of the same life table.

This chapter is concerned with a wide generalization of these familiar quantities, a generalization consisting in simultaneous consideration of many variables. The married couple risk not only divorce but childbearing, death, migration, and other contingen- 
cies-and they risk them all at each moment. Within any one of these broad groups of contingencies are distinctions: People not only risk death but they risk death by motor vehicle accident, cancer, and heart failure. The various causes of death are not independent; if a man dies of heart disease in the middle of the year, he is not eligible to die in an automobile accident in the latter half of the year. All the contingencies mentioned interact with others in this sense.

The purpose of this exposition is to show that combinations of great complexity, taking account simultaneously of death, migration, working and nonworking, and other movements, can be handled by formulas that are as simple as those for making an ordinary life table. Matrix algebra is in effect a computing device that keeps the combinations straight. Formulas that are multidimensional analogs of the ordinary life table can capture relations complex enough to discourage anyone who tried to keep them straight in his head.

The works of Fix and Neyman (1951), Mertens (1965), Sverdrup (1965), Chiang (1960a, 1960b, 1961, 1968), Oechsli (1971), Schoen and Nelson (1974), Schoen (1975), Hoem (1975), Hoem and Fong (1976), Schoen and Land (1978), and especially of Rogers (1975), Willekens (1978a), and Rogers and Ledent (1976) have resulted in a simple and precise way of handling these multivariate problems.

\section{SINGLE DECREMENT AS A MODEL}

Early in the history of demography two questions were asked: What is the probability of surviving to age $x$, and what is the average age at death-the expectation of life? Smith and Keyfitz (1977) provide excerpts from original papers. The answer, in current notation, is that if the chance of dying between age $a$ and $a+d a$ for those aged $a$ is $\mu(a) d a, \mu(a)$ being the force or intensity of mortality, then the probability $l(x)$ of survival to age $x$ is obtainable by solving the differential equation

$$
d l(x) / d x=-\mu(x) l(x)
$$


which gives $l(x)=\exp \left(-\int_{0}^{x} \mu(a) d a\right)$, and the expectation of life is then calculated as

$$
\stackrel{\circ}{e}(x)=\int_{x}^{\omega} l(a) d a / l(x)
$$

where $\omega$ is the oldest age of life.

When births are taken into account as well as deaths, an important question arises: By how many girls will a girl baby be replaced? The answer is given in terms of the chance $l(x)$ that she will live to age $x$ and then have a girl baby between age $x$ and $x+d x, m(x) l(x) d x$, where $m(x)$ is the age-specific fertility rate. Integrating this over the range of reproductive life gives the required $R_{0}=\int_{\alpha}^{\beta} m(x) l(x) d x$, which is the ratio of the size of one generation to the preceding at the specified rates of birth and death. It gives the implication for population growth of the prevailing schedule of mortality and fertility, in disregard of peculiarities of the existing age distribution.

These are just about the most complicated demographic problems that can be presented and solved in one dimension. Everything else requires two or more dimensions. To study mortality by itself, or even mortality and fertility, recognizing age only, is to take a very small part of the demographic process out of its natural context. What follows will generalize the preceding formulas to an arbitrary number of dimensions.

\section{MATRIX NOTATION}

To generalize the original life table theory, we deal not only with the movement from life to death represented by the scalar rate $\mu(x)$ but with the matrix $\mu(x)$, standing for the instantaneous rates of movement between states that are to be incorporated in the model-between life and death; between any pair of regions of a country; between work and unemployment; between being married and being divorced; between blue-collar and white-collar work. Crosses of one of these classifications with another can be recognized - for example, going from being married and out of the labor force to being divorced and working in an office. Once the basic 
rates (or forces or intensities) of movement for the $\boldsymbol{\mu}(x)$ matrix are known, all else can be found: the chance that a man of 30 who is married will be alive 10 years later and divorced, or the chance that a blue-collar person of 25 will be alive and doing white-collar work 30 years later. As in all demography, the reference is not so much to predict as to find the implications of a set of past rates.

Construction of the $\boldsymbol{\mu}(x)$ matrix from actual data, at least to a suitable approximation, is straightforward. The off-diagonal elements of $\boldsymbol{\mu}(x)$ are each the corresponding observed rate of movement in a small time interval with sign reversed. Thus $-\mu_{i j}(x) d x$ is minus the chance that a person in state $j$ transfers to state $i$ during the short period of time and age $d x$. Each diagonal element of $\boldsymbol{\mu}(x)$ contains the rate $\mu_{\delta i}$ of dying, with positive sign, along with the total of the off-diagonal elements of the column, $\Sigma_{i \neq j} \mu_{i j}$, also with positive sign. The reason for this is that the column total has to be conservative - that is, to add to zero with respect to movements among units. The quantities from the $j$ th state added into the $i$ th state must also be subtracted from the $j$ th, so an increment to $\mu_{i j}$, $i \neq j$, has to be subtracted from $\mu_{j j}$. The net total of the column is only the death rate.

The notation is indicated in Table 1 , giving the matrix $\boldsymbol{\mu}(x)$ in some detail. The right-hand subscript is state of origin, the left-hand subscript state of destination. Thus $\mu_{23}(x)$ is the movement from state 3 to state 2 for persons aged $x$. All other matrices use the same subscripting, essentially that of Rogers (1975). The matrix $\mathbf{M}(x)$ will be the finite approximation to $\boldsymbol{\mu}(x)$.

TABLE 1

Matrix $\boldsymbol{\mu}(x)$ of Moves

$$
\boldsymbol{\mu}(x)=\left[\begin{array}{cccc}
\mu_{\delta 1}(x)+\sum_{i \neq 1}^{n} \mu_{i 1}(x) & -\mu_{12}(x) & -\mu_{13}(x) & \cdots \\
-\mu_{21}(x) & \mu_{\delta 2}(x)+\sum_{i \neq 2}^{n} \mu_{i 2}(x) & -\mu_{23}(x) & \cdots \\
-\mu_{31}(x) & -\mu_{32}(x) & \mu_{\delta 3}(x)+\sum_{i \neq 3}^{n} \mu_{i 3}(x) & \cdots \\
\vdots & \vdots & \vdots & \vdots
\end{array}\right]
$$


Throughout we shall make the one major assumption that is common to all life tables and to increment-decrement tables and without which demographic processes cannot be conveniently portrayed. The probability of an individual making any transition will be taken to depend only on the state in which he is located at the start of the transition period-the Markov condition. The chance of a man of 55 dying before the age of 60 in the ordinary life table depends only on the fact that he is 55 and belongs to a certain defined population; it does not depend on his health as a baby or whether he smokes or is a nonsmoker or whether his father died young or old. If we want to take into account anything beside his age at the beginning of the interval, we have to do so by dividing up the population-say into smokers and nonsmokers-and then allowing the same Markov condition to apply within each of these groups.

Researchers in some fields find this assumption more restrictive than those in other fields. There is not much complaint about it in the ordinary life table. On the other hand, for mobility studies the history of the individual does seem to be important-for example, the longer a person has been in a given region, the less likely he is to move away in the next time interval. Such considerations introduce the history of the person in a way that precludes the treatment of these pages. We shall always assume that the entire history of the person is summed up in the state in which he is found at the beginning of each interval.

\section{THE KOLMOGOROV EQUATION}

Identical with Equation (1), except that the elements are now matrices and vectors, is the basic

$$
d\{l(x)\} / d x=-\mu(x)\{l(x)\}
$$

which is due originally in this application to Kolmogorov (Krishnamoorthy, 1978; Willekens, 1978a). Here $\{\boldsymbol{l}(x)\}$ is a vertical vector array in which the $i$ th element is the number of the population surviving and in the $i$ th category at age $x$. In general, where people are going in and out of the several categories, we cannot say that the elements of $\{l(x)\}$ represent probabilities, yet probabilities 
are what we seek. Now suppose that in the small interval of time and age $d x$ no one will be affected by more than one event. We would like to pass from $\boldsymbol{\mu}(x)$ and the vector $\{\boldsymbol{l}(x)\}$ to a matrix $\boldsymbol{l}(x)$ whose typical element is $l_{i j}(x)$, the chance that a person born in the $j$ th state will be in the $i$ th state by age $x$.

The theory for doing this is available from standard works on linear differential equations (Coddington and Levinson, 1955; Gantmacher, 1959, vol. 2, p. 113). If there are $n$ states, and so the matrix $\mu(x)$ is $n \times n$, and if the $n$ latent roots of that matrix are distinct, then there will be $n$ linearly independent vectors $\{l(x)\}$ that satisfy Equation (3). When this is so, the matrix made by setting those vectors side by side will obviously also satisfy the equation, and it can be shown to be the complete solution. Call $l(x)$ the matrix made up of the several $\{l(x)\}$. We shall see how to obtain the elements of $\boldsymbol{l}(x)$ so as to ensure that the $i j$ th element is the probability that a person born in the $j$ th category finds himself in the $i$ th category by age $x$. The procedure is due to Rogers (1975).

\section{THE MULTIPLICATIVE PROPERTY}

One mathematical property of the $l(x)$ will be important for the demographic application: its multiplicativity. It may be shown (though not here) that if the interval from zero to $y$ is broken into two subintervals at any point, say $x<y$, then (Gantmacher, 1959, vol. 2, p. 127)

$$
l(y)=l(y \mid x) l(x)
$$

where the $i j$ th element of $l(y \mid x)$ will in our interpretation mean the probability of being in the $i$ th state at age $y$, given that the person was in the $j$ th state at age $x$. Since the interval from $x$ to $y$ may also be split into subintervals with the same property, we can divide the whole of any range into sufficiently small intervals (usually 1 or 5 years) that within each interval $\boldsymbol{\mu}(x)$ may be approximated by a matrix whose elements are constants independent of age. This will be the key to the numerical solution of Equation (3).

What we cannot do is calculate directly the exponential of minus the integral of $\boldsymbol{\mu}(x)$, in analogy to what is possible for the one-region life table solution of Equation (1). The exponential of an 
integral has meaning only when the matrix commutes. The relation of exponentials $e^{A+B}=e^{A} \times e^{B}$ requires commutativity, as a fortior does $e^{\int A(x) d x}$. Only diagonal matrices and other uninteresting special cases are commutative. We must break down the interval from zero to $x$ into subintervals short enough, say $h$ in length, that the $\mu_{i j}(x)$ may be taken as constant within each of them. If in the interval $x$, $x+h, \mu_{i j}(x)$ is constant, say $M_{i j}$, for all $i$ and $j$, and $\mathbf{M}_{x}$ is the array of the $M_{i j}$, then from property (4) we can write

$$
l(x+h)=e^{-h \mathbf{M}_{x}} \boldsymbol{l}(x)
$$

With an arbitrary radix $l(0)$, Equation (5) permits the construction of $l(x)$ step by step at intervals of $h$ all the way to the end of life. Alternatively, expanding the exponential in (5) to its first two terms gives

$$
\boldsymbol{l}(x+h) \doteq\left(\mathbf{I}-h \mathbf{M}_{x}\right) \boldsymbol{l}(x)
$$

This approximation can be improved by first premultiplying Equation (5) on both sides by $\exp \left(h \mathbf{M}_{x} / 2\right)$ and then expanding to obtain the more symmetric

$$
\left(\mathbf{I}+h \mathbf{M}_{x} / 2\right) l(x+h)=\left(\mathbf{I}-h \mathbf{M}_{x} / 2\right) l(x)
$$

or on multiplying by $\left(\mathbf{I}+h \mathbf{M}_{x} / 2\right)^{-1}$ on the left,

$$
\boldsymbol{l}(x+h)=\left(\mathbf{I}+h \mathbf{M}_{x} / 2\right)^{-1}\left(\mathbf{I}-h \mathbf{M}_{x} / 2\right) \boldsymbol{l}(x)
$$

Thus Equation (5) is an approximation to (4) for $y-x=h$, and (6) and (7) are approximations to (5). The approximation (7) is close enough for many kinds of data with intervals of 1 year or even 5 years. It can always be improved by graduating the original data down to tenths of a year or smaller, and this was essentially what Oechsli $(1971,1975)$ did, using spline functions.

It is possible to escape from the restriction of Equations (5) and (7) that the rates be constant over the interval $h$. Willekens (1978a) and Krishnamoorthy (1978) have done this by using the Volterra theory of integration. As a consequence of (3),

$$
\begin{aligned}
\boldsymbol{l}(x+h) & =\boldsymbol{l}(x)-\int_{x}^{x+h} \boldsymbol{\mu}(t) \boldsymbol{l}(t) d t \\
& =\left[\mathbf{I}-\int_{x}^{x+h} \boldsymbol{\mu}(t) \boldsymbol{l}(t) \boldsymbol{l}^{-1}(x) d t\right] l(x)
\end{aligned}
$$


With observed or appropriately constructed curves for $\mu(t)$ and $l(t)$ within the interval $h$, the square bracket can be evaluated.

A third approach is due to Schoen and Land (1978). They obtain flow equations, the multidimensional analog to $l_{x+h}=$ $l_{x}-{ }_{h} d_{x}$, representing relations within the life table. Alongside these are orientation equations, analogous to ${ }_{h} M_{x}={ }_{h} d_{x} /{ }_{h} L_{x}$, where ${ }_{h} M_{x}$ is the observed rate. Finally the set is completed with numerical integration equations analogous to ${ }_{h} L_{x}=(h / 2)\left(l_{x}+l_{x+h}\right)$. As in the single-region case, the solution can be given explicitly with a straight-line integration formula. With more elaborate integration formulas iteration is required.

The initial $l(0)$ is arbitrary as far as the differential equation (3) is concerned; we shall define it as the unit matrix I. In instances where a population model is to be constructed rather than a set of probabilities, so that radices other than $\mathbf{I}$ are required, those will be entered by multiplication: $l(x) \mathbf{Q}$, where $\mathbf{Q}$ is a diagonal matrix containing the starting numbers or births in the several categories recognized.

\section{PROBABILITIES OVER LONG INTERVALS}

The most obvious question to ask is: What is the probability that a person in the $j$ th state at age $x$ will find himself in the $i$ th state at age $y$, where the difference $y-x$ need not be small? Without matrix methods the problem is difficult and has even been thought unsolvable. It has to take account not only of movement out of the $j$ th state but also of movement into the $i$ th state of persons not in the $j$ th state at age $x$. It may be solved by the multiplicative property referred to above as applicable wherever the interval $(x, y)$ can be broken down into subintervals of width $h$, within each of which $l(x+h) l^{-1}(x)$ can be calculated by any of the methods cited earlier. If $l(y \mid x)$ is the desired set of probabilities, we know that $l(y \mid x) l(x)=l(y)$, so multiplying on the right by $l^{-1}(x)$ we get

$$
l(y \mid x)=l(y) l^{-1}(x)
$$

where the probability of going from the $j$ th state at age $x$ to the $i$ th state at age $y>x$ is the $j$ th element of the $i$ th row of $l(y \mid x)$. 
It can be argued that the differential equation (3) is a background of mathematical theory used only to provide a context for our symbols far more general than will be called for by demographic applications. Why could we not be satisfied to build up the $l(x)$ matrix from the $\mathbf{M}_{x}$ matrix, step by step, in 5-year age groups starting from the unit matrix $l(0)=I$, using Equation (7) at each step? We could even go through the arithmetic and obtain every probability required without ever introducing matrix notation, but the process of tracing individual combinations would be tedious. Moreover, the general theory assures us of the multiplicativity of the $l(x)$ matrix, in the sense of Equation (4), and from this all else follows.

\section{EXPECTED TIME IN THE SEVERAL STATES}

Beyond probabilities we would like to know the expected time lived between age $x$ and $x+h$ in the several states, where in the first instance $h$ is small. A straight-line approximation gives as time of residence in the $i$ th state for those initially in the $j$ th state the matrix

$$
{ }_{h} \mathrm{~L}_{x}=(h / 2)\left(l_{x}+l_{x+h}\right)
$$

and a cubic gives

$$
{ }_{h} \mathbf{L}_{x}=(13 h / 24)\left(l_{x}+l_{x+h}\right)-(h / 24)\left(l_{x-h}+l_{x+2 h}\right)
$$

Adding ${ }_{h} \mathbf{L}_{x}$ gives the person-years over any interval of age large or small. Cumulating ${ }_{n} \mathbf{L}_{x}$ back from the end of the table gives the expected years in the $i$ th state from age $x$ to the end of life measured prospectively from birth in the $j$ th state:

$$
\mathbf{T}(x)=\int_{x}^{\omega-h} \mathbf{L}(a)=\int_{x}^{\omega} l(a) d a
$$

For an individual just born in the $j$ th state, the probability of being in the $i$ th state by age $x$ is the $i j$ th element of $l(x)$. And if the expected number of years beyond age $x$ in the $k$ th state for those who survive to the $i$ th state by age $x$ is the $i k$ th element of $\stackrel{\mathrm{e}}{(x)}$ we must have

$$
\mathbf{T}(x)=\stackrel{\circ}{ }(x) l(x)
$$


where the right-hand side gives for the $j$ th state at birth the number of years that can be collected if one reaches age $x$ (and is then in the $i$ th state) times the probability of reaching the $i$ th state by age $x$.

Consider, for example, those in the second state at birth and let us find their expectation beyond age $x$ in the first state. The second column of $l(x)$ gives the chance that the person born in the second state is in the first, the second, and so forth, state at age $x$ : If residing in the first state at age $x$, he has an expected $\stackrel{\circ}{11}_{11}(x)$ in the first state; if residing in the second, he has an expected $\dot{e}_{12}(x)$ in the first; and so on. In short his total expectation in the first state, given that he was born in the second, is prospectively from age zero

$$
\stackrel{\circ}{11}_{1}(x) l_{12}(x)+\stackrel{\circ}{12}_{12}(x) l_{22}(x)+\stackrel{\circ}{13}_{13}(x) l_{32}(x)+\cdots
$$

For the whole collection of states, we have

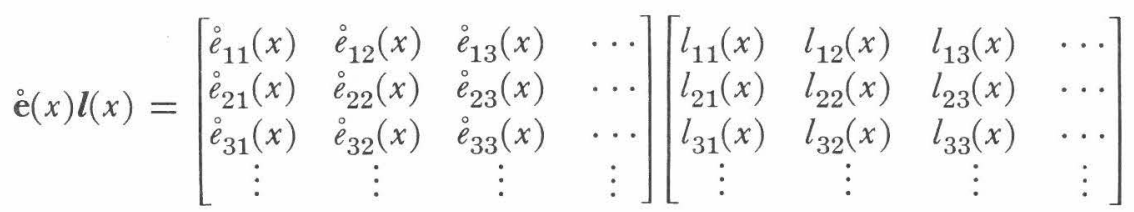

Note that here as in other expressions indexes are read right to left in order to use column vectors and the conventional subscripting of matrix elements.

Dividing Equation (10) by $l(x)$ on the right, we have for the expectation in the $i$ th state for a person in the $j$ th state at age $x$ the ij th element of

$$
\stackrel{\circ}{\mathbf{e}}(x)=\mathbf{T}(x) \mathbf{l}^{-1}(x)
$$

An example is Table 2, calculated by Frans Willekens from 1967-1972 United States data covering both sexes combined. It shows a total expectation of life of 71.08 years for those born in the Northeast. Of this time they will spend 13.16 years in the South. On the other hand, those born in the South will spend only 7.73 years in the Northeast, all on the average and provided that the rates of the given period, 1967-1972, continue to apply. As Willekens and Rogers (1976, p. 30) say: "The multiregional life table decomposes the expectation of life according to where that life is spent. It introduces the spatial dimension into classical demographic analysis." 
TABLE 2

Life Expectancies at Birth by Region, Both Sexes Together, United States: 1967-1972

Place of Birth

$\begin{array}{lrrrr}\quad \begin{array}{c}\text { Place of } \\ \text { Residence }\end{array} & \begin{array}{c}\text { North- } \\ \text { east }\end{array} & \begin{array}{c}\text { North } \\ \text { Central }\end{array} & \text { South } & \text { West } \\ \text { Northeast } & 41.73 & 5.84 & 7.73 & 6.57 \\ \text { North Central } & 8.19 & 39.89 & 11.95 & 11.64 \\ \text { South } & 13.16 & 14.69 & 39.52 & 15.24 \\ \text { West } & 8.01 & 10.66 & 11.30 & 37.70 \\ \text { Total } & 71.08 & 71.08 & 70.50 & 71.15\end{array}$

SOURCE: Calculated by Frans Willekens.

Note that, on the definitions provided, the $i j$ th element of $\mathbf{T}(x)$ is the average length of time spent in the $i$ th state by those born in the $j$ th state. For $\mathbf{T}(0)$ birth and initial residence are the same; but for any later $\mathbf{T}(x)$ they are different and give rise to two different expectations. Dividing on the right by $l^{-1}(x)$, as was done in Equation (11), provides the expectations of stay in the $i$ th state for each $j$ th state of residence at age $x$.

To find the expected stay in the $i$ th state for each state of birth requires a different denominator. The chance that the person born in state $j$ is in state $i$ at age $x$ is $l_{i j}(x)$; the total $\Sigma_{i} l_{i j}(x)$ of the $j$ th column of this through all $i$ gives the chance that the person born in state $j$ is still alive at age $x$, irrespective of where he lives at that time. If the diagonal matrix of these totals is called $\bar{l}(x)$, then we have for the expectations $\overline{\mathrm{e}}(x)=\mathrm{T}(x) \bar{l}^{-1}(x)$. Region of residence at age $x$ has been duly summed out.

The multigroup life table often imposes distinctions not required in the ordinary life table.

\section{FERTILITY EXPECTATIONS}

Rogers (1975, p. 106) and Willekens and Rogers (1977) go on to discuss fertility expectations based on the same data, now relating births to total population age by age. The result is in effect an average of the male and female net reproduction rates. Whether for females alone or for both sexes, we can write the age-specific 
birth rate in the $i$ th region over a short time interval $d x$ as $\nu_{i}(x)$, construct the diagonal matrix $\boldsymbol{\nu}(x)$, and postmultiply by $\boldsymbol{l}(x)$ :

$$
\boldsymbol{\nu}(x) \boldsymbol{l}(x)=\left[\begin{array}{cccc}
\nu_{1}(x) & 0 & \ldots & \cdots \\
0 & \nu_{2}(x) & \ldots & \ldots \\
\vdots & \vdots & \vdots & \vdots
\end{array}\right]\left[\begin{array}{cccc}
l_{11}(x) & l_{12}(x) & \ldots & \cdots \\
l_{21}(x) & l_{22}(x) & \ldots & \ldots \\
\vdots & \vdots & \vdots & \vdots
\end{array}\right]
$$

to obtain

$$
\left[\begin{array}{cccc}
\nu_{1}(x) l_{11}(x) & \nu_{1}(x) l_{12}(x) & \cdots & \cdots \\
\nu_{2}(x) l_{21}(x) & \nu_{2}(x) l_{22}(x) & \cdots & \cdots \\
\vdots & \vdots & \vdots & \vdots
\end{array}\right]
$$

whose $i j$ th element gives the probability that a person (or woman) born in the $j$ th region gives birth to a child (or girl child) in the $i$ th region. When this is integrated over all $x$ we have the multiregional net reproduction rate $\mathbf{R}_{0}: \mathbf{R}_{0}=\int_{\alpha}^{\beta} \boldsymbol{\nu}(x) \boldsymbol{l}(x) d x$.

That $\mathbf{R}_{0}$ is shown in Table 3 for the United States (19671972). The child born in the Northeast could expect to have 1.19 babies on the average. Of these only 0.74 would be born in the Northeast; 0.13 would be born in the North Central region, and so forth. The table shows the implications of the data about 1970 for the birthplaces of successive generations, averaging the sexes.

TABLE 3

Fertility Expectancies by Region, Both Sexes Together, United States: 1967-1972

Place of Birth

$\begin{array}{lcccc}\begin{array}{l}\text { Place of } \\ \text { Residence }\end{array} & \begin{array}{c}\text { North- } \\ \text { east }\end{array} & \begin{array}{c}\text { North } \\ \text { Central }\end{array} & \text { South } & \text { West } \\ \text { Northeast } & 0.74 & 0.09 & 0.13 & 0.10 \\ \text { North Central } & 0.13 & 0.74 & 0.21 & 0.20 \\ \text { South } & 0.20 & 0.23 & 0.70 & 0.24 \\ \text { West } & 0.12 & 0.16 & 0.18 & 0.65 \\ \text { Total } & 1.19 & 1.22 & 1.22 & 1.19\end{array}$

SOURCE: Calculated by Frans Willekens. 


\section{MARRIAGE TABLES}

Krishnamoorthy (1978) has presented a decrement table for women by marital status, recognizing the states of single, married, widowed, and divorced and applying United States data of 1970. He works from the observed transition rates to the $l(x)$ and finds, for instance, that the chance of a child just born being in single (never married) status by age 50 is 0.034 ; of being married 0.727 ; of being widowed (and not remarried) 0.067; and of being divorced (and not remarried) 0.090. At birth, expected number of years single is 22.56; married (including remarriages) 32.27; widowed 10.22; divorced 4.71. Demographers have always recognized that the proper way to look at rates of divorce, for example, is to produce such a life table. The methods described here provide a simple and accurate mechanism for making such life tables.

\section{PROJECTION}

A common demographic activity is population projection. If a given set of probabilities applies, and we know the vector representing the several categories of population at a certain point of time, the expected numbers at a later point of time are calculable. Suppose the age interval as well as the time interval to be $h$ years as before; then for a population that happened to be concentrated at ages $0, h, 2 h, \ldots$, the matrix $l(x+h) l^{-1}(x)$ would be appropriate for the projection in all cases where $l(x)$ is nonsingular. But to approximate the observed population by a series of spikes seems inferior to approximation by a series of histograms.

We need a ratio corresponding to $S_{x}={ }_{h} L_{x+h} /{ }_{h} L_{x}$ of the single-region case. This is obtained by applying the multiplicative property (4) to show that $\mathbf{L}_{x+h}=\mathbf{S}_{x} \mathbf{L}_{x}$, and multiplying on the right by $\mathbf{L}_{x}^{-1}$ to obtain $\mathbf{S}_{x}=\mathbf{L}_{x+h} \mathbf{L}_{x}^{-1}$, again assuming the inverse of $\mathbf{L}_{x}$ exists. (It does not exist for a marriage or labor force table prior to age 15 or so, and some other device is needed.) Now suppose the transitions pertaining to each given age, perhaps transitions among regions, are assembled into a block. Such blocks for the several ages $x$ may be assembled into a matrix $\mathbf{S}$ with zero blocks everywhere 
except in the subdiagonal. The arbitrary (that is, observed) $\left\{\mathbf{K}_{t}\right\}$ is projected to time $t+h$ by

$$
\left\{\mathbf{K}_{t+h}\right\}=\mathbf{S}\left\{\mathbf{K}_{t}\right\}
$$

This projects the part of the population already alive, but it gives no attention to births. To allow for them we need a matrix $\mathbf{F}$, whose nonzero elements are in its first row of submatrices, so the complete projection with time-invariant coefficients is (Rogers, 1975; Feeney, 1970)

$$
\left\{\mathbf{K}_{t+h}\right\}=(\mathbf{S}+\mathbf{F})\left\{\mathbf{K}_{t}\right\}
$$

The procedure described by (13) permits a certain kind of experimentation with the elements of $\mathbf{S}$ and $\mathbf{F}$ and serves to ascertain the effects on future population of marriage ages, migration rates from more to less fertile regions, and so on. For forecasting purposes, some gains can be obtained by deliberately allowing the coefficients to change according to what one thinks may be the trends over future time. One must be cautious here; in more than one case varying the coefficients has produced a less accurate forecast than fixed coefficients would have done.

\section{TRANSITION VS. INSTANTANEOUS PROBABILITY OF MOVING}

Most data on geographical mobility come in the form of transitions over a finite period-we know that the person was in state $j$ at one moment of time and in state $i 1$ year or 5 years later. The census, for instance, asks people where they were 5 years ago without inquiring about intermediate moves. We shall speak of transitions in terms of a $\mathbf{P}$ matrix, whose $p_{i j}$ is the probability that a person is in the $j$ th state this year and in the $i$ th state 1 year or 5 years later, in contrast to the $\boldsymbol{\mu}$ matrix, where the chance of moving from the $j$ th state to the $i$ th in time $d x$ is $\mu_{i j}(x) d x$.

Although our theory started out in terms of movements in time separated by an infinitesimal interval $d x$, it then dropped the $\mu$

and took no account of what happens within the finite interval beyond the probability that a person in the $j$ th state at the begin- 
ning is in the $i$ th state at the end. This is satisfactory if the time interval is short enough that two moves do not occur within it - that a person does not move and die in the same specified $h$ years, for example. But what if the data cover a wide enough interval that several moves are possible?

Fortunately, the bias does not apply to most of the quantities calculated from the model. For projecting population according to the spike representation of the $l(x)$, the observed transitions are obviously the right ones to use; one wants to omit intermediate transitions (people going from $j$ to $i$ and back again, all within one time interval) in the future as they have been omitted in the past. Slightly less obviously, the same applies to projection by histograms (Equation 13). On the other hand, the expected number of moves calculated from the model will be understated if multiple moves occurring within the unit time interval of the data are neglected.

The Markov assumptions of our model (without which none of the manipulations of this article would be possible) assign the same probabilities to everyone in a given state at the beginning of each interval; no past history is allowed to influence the chance of transition beyond what is implied by the state that the person is then occupying. If we suppose this also applies in all subintervals, then the Markov assumption allows us to capture and add in those jumps that occur within intervals.

Consider transitions of one period between two states, in which $p_{i j}=p_{j i}=p$, so that the fraction of persons initially in the $j$ th state that are found in the $i$ th state at the end of the unit period is $p$. Suppose also that the instantaneous probability of moving (not known) in the time $d t$ is $\mu d t$; then we can write out in terms of $\mu$ what fraction of the individuals initially in the $j$ th state would be found in the $i$ th state at the end of the period. It is those who made an odd number of moves, 1 or 3 or 5 or 7 , this being as far as we need to go. If the instantaneous rate is $\mu$, then by integration the probability of one transition in unit time is $\mu e^{-\mu}$, the probability of three transitions is $\left(\mu^{3} / 3 !\right) e^{-\mu}$, and so on, and the sum of these can be equated to $p$ :

$$
\begin{aligned}
p & =\mu e^{-\mu}+\left(\mu^{3} / 3 !\right) e^{-\mu}+\cdots \\
& =\left(e^{-\mu} / 2\right)\left(e^{\mu}-e^{-\mu}\right)=\left(1-e^{-2 \mu}\right) / 2
\end{aligned}
$$


Solving for $\mu$ gives

$$
\begin{aligned}
1-2 p & =e^{-2 \mu} \\
\mu & =-\frac{1}{2} \ln (1-2 p)
\end{aligned}
$$

(I am grateful to Jan Hoem for this form of the solution.)

Table 4 gives values of $p$ and the corresponding values of $\mu$. It says, for example, that if the transition probability from the $j$ th to the $i$ th state is 0.2 , and the transition probability from the $i$ th to the $j$ th is the same as this, and there are no other states, then an instantaneous rate of 0.255 will produce the 0.200 chance of transition. There will be a probability $e^{-\mu}=0.775$ of no moves, $\mu e^{-\mu}=0.198$ of one move, $\left(\mu^{2} / 2 !\right) e^{-\mu}=0.025$ of two moves, and so on.

To generalize, if $p_{i j}$ is the probability of a transition from state $j$ to state $i$ in unit time, we would like to find the instantaneous probability $\mu_{i j}$ (fixed over the interval) that would provide gross movement but still show a net of $p_{i j}$. We have discussed only the simple case where $p_{i j}=p_{j i}=p$ is the probability of transition in either direction between the two states.

The generalization that follows from the Kolmogorov equation (Kitsul, 1978) is the matrix relation $\mathbf{P}=\exp (-\boldsymbol{\mu})$, or written out:

$$
\left[\begin{array}{cccc}
p_{11} & p_{12} & \cdots & \cdots \\
p_{21} & p_{22} & \cdots & \cdots \\
\vdots & \vdots & \vdots & \vdots
\end{array}\right]=\exp \left(-\left[\begin{array}{cccc}
\mu_{11} & \mu_{12} & \cdots & \cdots \\
\mu_{21} & \mu_{22} & \cdots & \cdots \\
\vdots & \vdots & \vdots & \vdots
\end{array}\right]\right)
$$

where the diagonals $\mu_{i i}$ are such as to make the columns sum to zero. If the matrices have distinct roots, (15) may be solved numerically in either direction.

Let us prove that (14) is a special case of (15). Take the matrices as $2 \times 2$ and make the $\mu$ 's equal to $\mu$ or $-\mu$, so that (15) becomes

$$
\left[\begin{array}{ll}
p_{11} & p_{12} \\
p_{21} & p_{22}
\end{array}\right]=\exp \left(-\left[\begin{array}{rr}
\mu & -\mu \\
-\mu & \mu
\end{array}\right]\right)
$$

To evaluate the right-hand side, we need eigenvalues, eigenvectors, 
TABLE 4

Transition Probability $p$, Corresponding Instantaneous Rate $\mu$, and Probability of $1,2, \ldots, 5$ Moves in Two-State Model with $P_{i j}=P_{j i}=p$

\begin{tabular}{|c|c|c|c|c|c|c|c|}
\hline \multirow{2}{*}{$\begin{array}{l}\text { Transition } \\
\text { Probability } \\
(p)\end{array}$} & \multirow{2}{*}{$\begin{array}{c}\text { Corresponding } \\
\text { Instantaneous } \\
\text { Rate } \\
(\mu)\end{array}$} & \multicolumn{6}{|c|}{ Probability Of } \\
\hline & & 0 Moves & 1 Move & 2 Moves & 3 Moves & 4 Moves & 5 Moves \\
\hline 0.001 & 0.001 & 0.999 & 0.001 & 0.000 & 0.000 & 0.000 & 0.000 \\
\hline 0.050 & 0.053 & 0.949 & 0.050 & 0.001 & 0.000 & 0.000 & 0.000 \\
\hline 0.100 & 0.112 & 0.894 & 0.100 & 0.006 & 0.000 & 0.000 & 0.000 \\
\hline 0.150 & 0.178 & 0.837 & 0.149 & 0.013 & 0.001 & 0.000 & 0.000 \\
\hline 0.200 & 0.255 & 0.775 & 0.198 & 0.025 & 0.002 & 0.000 & 0.000 \\
\hline 0.250 & 0.347 & 0.707 & 0.245 & 0.042 & 0.005 & 0.000 & 0.000 \\
\hline 0.300 & 0.458 & 0.632 & 0.290 & 0.066 & 0.010 & 0.001 & 0.000 \\
\hline 0.350 & 0.602 & 0.548 & 0.330 & 0.099 & 0.020 & 0.003 & 0.000 \\
\hline
\end{tabular}

SOURCE: Calculated by Frans Willekens. 
and spectral components. For eigenvalues,

$$
\left|\begin{array}{cc}
\mu-\lambda & -\mu \\
-\mu & \mu-\lambda
\end{array}\right|=0
$$

or

$$
\mu^{2}-2 \lambda \mu+\lambda^{2}-\mu^{2}=0
$$

Therefore $\lambda=0$ or $2 \mu$. Left eigenvectors are [1 1 1 ] and [ $\left.\begin{array}{ll}1 & -1\end{array}\right]$, up to constant factors. Right eigenvectors are $\left\{\begin{array}{r}1 \\ -1\end{array}\right\}$ and $\left\{\begin{array}{r}1 \\ -1\end{array}\right\}$. Spectral components $\mathbf{Z}_{1}$ and $\mathbf{Z}_{2}$ are $\frac{1}{2}\left[\begin{array}{ll}1 & 1 \\ 1 & 1\end{array}\right]$ and $\frac{1}{2}\left[\begin{array}{rr}1 & -1 \\ -1 & 1\end{array}\right]$. Hence

$$
\exp (-\boldsymbol{\mu})=\left(e^{0} / 2\right)\left[\begin{array}{ll}
1 & 1 \\
1 & 1
\end{array}\right]+\left(e^{-2 \mu} / 2\right)\left[\begin{array}{rr}
1 & -1 \\
-1 & 1
\end{array}\right]
$$

Multiply by $e^{-\mu} e^{\mu}$ to obtain as the $\mathbf{P}$ matrix

$$
\left(e^{-\mu} / 2\right)\left\{e^{\mu[}\left[\begin{array}{ll}
1 & 1 \\
1 & 1
\end{array}\right]+e^{-\mu}\left[\begin{array}{rr}
1 & -1 \\
-1 & 1
\end{array}\right]\right\}
$$

The lower left element of this is

$$
e^{-\mu}\left[\left(e^{\mu}-e^{-\mu}\right) / 2\right]=e^{-\mu}\left(\mu+\mu^{3} / 3 !+\mu^{5} / 5 !+\cdots\right)
$$

which is identical with (14).

Equation (15) allows us to infer the instantaneous probabilities of movement that are equivalent to a given set of transitions and hence to know the multiple transitions expected in any finite period. This is based on the Poisson process in which the probability of moving from the $j$ th to the $i$ th state is equal for all moments within the unit time period. In the case of death, a movement is the same as a transition and no reversal is possible; for other changes, transition in either direction has to be admitted. Under the stated assumptions, Equation (15) read as an equation in $\mathbf{P}$ tells the transitions that correspond to a given set of movement probabilities; read as an equation in $\mu$, it tells the movement probabilities that account for the given transitions.

In a series of papers Singer and Spilerman (1976) have shown how to find the continuous-time Markov chain of which an observed set of transitions can be considered the manifestation. Not 
every series of transitions is embeddable in a stationary Markov chain (that is, one with fixed parameters). They represent the solution of $\mathrm{p}=e^{-\mu}$ formally as $\boldsymbol{\mu}=-\ln \mathrm{p}$ and proceed to create a suitable definition of $\ln \mathbf{p}$ in terms of the decomposition of $\mathbf{p}$ in spectral components. Because the logarithm has multiple branches, the result is not unique, and identifying the answer that corresponds to the problem in hand requires some ingenuity. The reader is referred to Singer and Spilerman for a highly sophisticated treatment of the relation between $\mathrm{p}$ and $\boldsymbol{\mu}$.

\section{STABLE POPULATION}

When we seek probabilities and expected values for individuals, the radix is taken as $l(0)=\mathrm{I}$. But for stationary populations the radix must be the number of births into each of the categories. The point does not arise in tables of the single and married population, nor of the working population or the school population, because for each of these classifications everyone starts out in the same state-single, not in the labor force, and not at school. But the classification by territory is different: Portrayal of stationary conditions in the several regions requires that we enter as the radix a diagonal matrix $\mathbf{Q}$ showing the number of equivalent births in each region; here we use not $l(x)$ but $l(x) \mathbf{Q}$.

Like the usual single-region life table, our multigroup table is not only suited to provide probabilities and expectations but is also a population model for such groups as regions, occupations, marital statuses, or years of schooling. It gives the age and group distribution to which the age-group-specific rates would lead if they were in operation long enough that the peculiarities of the original distribution were forgotten.

But for this purpose the stationary model can readily be improved on by incorporating population increase. In the stable model the people now aged $x$ were born $x$ years ago when the population was smaller than it is now in the ratio $e^{-r x}$, so the number that would be counted as of age $x$ would be proportional to the survivors from one birth times $e^{-r x}$. On a radix of one birth in each region the stable multigroup population would show the age 
and region distribution $e^{-r x} l(x)$; on a basis of $q_{i}^{s}$ births in the $i$ th region it would show

$$
e^{-r x} l(x) \mathbf{Q}^{s}
$$

where $\mathbf{Q}^{s}$ is the diagonal matrix containing the $q_{i}^{s}$. The $j$ th element of the $i$ th row of $e^{-r x} l(x) \mathrm{Q}^{s}$ is $e^{-r x} l_{i j}(x) q_{j}^{s}$, which is the number of persons of age $x$ out of the $q_{j}^{s}$ born in the $j$ th region that will be found at stability in the $i$ th region. The $\mathbf{Q}^{s}$ is the multiregion analog of the stable equivalent.

Such a model mimics observed populations with a closeness that depends on how nearly constant is the regime of mortality and fertility to which they have been subject. The model in a sense tells the implication of present rates - what the outcome will be if they continue unchanged for two or three generations. It tells age by age where the Northeast would stand with respect to the rest of the United States if the age-specific rates of 1967-1972 continued to prevail.

The stable model gives a useful answer to the question of how prevalent is divorce. Alarm is expressed that the number of divorces in the United States is now approaching the number of first marriages, but the ratio of current divorces to current marriages is a poor measure of the seriousness of divorce. Much better is to use the expected proportion of marriages that will end in divorce-that is, the value of the relevant $l_{i j}$ in the multigroup table. But insofar as the population is increasing, the stable marital status-by-age table gives another answer: It tells what proportion of the population will be in the divorced condition at any one time. To make the model truly useful, we need a classification of the stable population into groups that are homogeneous in the propensity to divorce; it makes a great deal of difference for analysis whether one fifth of the population averages five divorces and the rest of the population none, or all of the population averages one divorce. The multigroup model lends itself well to a calculation by marital status, age, and propensity group, but unfortunately the data to enter in the model are lacking.

It is not to be expected that the multiregional table will have the same significance as the single-region table. After all, the ordinary life table is dominated by universal biological relations 
that are altered slowly, in particular the bathtub curve of deaths. Within any country the currents of migration have much less persistence. American migration has been steadily toward the sunbelt during the last one or two intercensal periods; prior to that it was northward from the cotton states. Such gross changes of direction deprive a stable representation of some of its interest. Thus one can only repeat and emphasize the caution that is also appropriate for the single-region table: Consider how closely the assumptions of the model fit past and prospective conditions. Use the model freely to say what will happen if present conditions persist; be cautious in assuming that they will persist.

\section{THE RENEWAL EQUATION}

So much for stationary and stable populations. This exposition has barely touched on self-renewing populations. We go on to the standard renewal process, but now extended (Rogers, 1975; Le Bras, 1971) to several regions or other groups. The multiregional renewal equation is written

$$
\{\mathbf{B}(t)\}=\{\mathbf{G}(t)\}+\int_{\alpha}^{\beta} \boldsymbol{\nu}(x) l(x)\{\mathbf{B}(t-x)\} d x
$$

where $\boldsymbol{\nu}(x)$ is a diagonal matrix whose $\nu_{i}(x)$ is the instantaneous birth probability at age $x$ in the $i$ th region; $b_{i}(t)$, the typical element of $\{\mathbf{B}(t)\}$, is the number of births in the $i$ th region; and $\{\mathbf{G}(t)\}$ is the number of births due to the population present at the initial time.

Equation (17) relates the births in the several regions at time $t$ to the births of the preceding generation. It does this region by region; it permits births now in region $i$ to be the daughters of births $x$ years ago in region $j$. The matrix $\boldsymbol{\nu}(x)$, containing birth rates by age and region of current residence, multiplies the survivorships $l(x)$, similarly classified; the product $\boldsymbol{\nu}(x) \boldsymbol{l}(x)=\boldsymbol{\phi}(x)$ is the multiregional net maternity function, whose integral has been designated $\mathbf{R}_{0}$. The function $\phi(x)$ multiplies the vertical vector of births in the preceding generation; the whole is integrated, added to $\{\mathbf{G}(t)\}$, and equated to current births.

As in the single-region case, the solution of the equation implies under very general conditions a fixed ultimate rate of 
increase of the system, as well as a varying rate that sooner or later converges to this fixed rate. The solution is found by trying $\{\mathbf{Q}\} e^{r t}$ in the homogeneous version $(\{\mathbf{G}(t)\} \equiv\{\mathbf{0}\})$ and so obtaining an equation in $r$ :

$$
\{\mathbf{Q}\}=\left[\int_{\alpha}^{\beta} e^{-r x} \boldsymbol{\nu}(x) \boldsymbol{l}(x) d x\right]\{\mathbf{Q}\}=\psi(r)\{\mathbf{Q}\}
$$

The problem here, as in the single-region case, reduces to finding a value of $r$ for which the several equations are consistent-that is, for which $[\psi(r)-\mathbf{I}]\{\mathbf{Q}\}=\{0\}$ can be solved. It can be solved for those values of $r$ for which the eigenvalue of $\psi(r)$ is unity - that is, for which the determinant $|\psi(r)-I|$ vanishes (Rogers, 1975, p. 94). Rogers develops the matrix analog of the single regional equation, including the use of Laplace transforms, to find the solution as proposed by Feller (1941). Willekens (1977) develops the multiregional reproductive value, again in surprisingly compact form, and shows how it leads to a simple expression for the ultimate stationary distribution when increase drops to zero.

Once such a model is set up it can be used for many kinds of sensitivity analysis. Willekens (1977) presents techniques of matrix differentiation and applies them to various demographic problems.

\section{CONTROL MODELS}

Willekens (1976a and 1978b) shows how policy variables can be introduced into the matrix model to provide simple solutions to the control problem. To the generalized Leslie model or state equation

$$
\left\{\mathbf{P}_{t+1}\right\}=\mathbf{G}\left\{\mathbf{P}_{t}\right\}
$$

we add a term that includes $\left\{\mathbf{Z}_{t}\right\}$, an input vector that incorporates exogenous policy variables, and $\mathbf{H}_{t}$, which gives the effect of the exogenous variables on $\left\{\mathbf{P}_{t+1}\right\}$ :

$$
\left\{\mathbf{P}_{t+\mathbf{1}}\right\}=\mathbf{G}\left\{\mathbf{P}_{t}\right\}+\mathbf{H}\left\{\mathbf{Z}_{t}\right\}
$$

Thus $h_{i j}$ is the effect on the $i$ th element of the state vector of a unit of the $j$ th control variable. Willekens shows how to solve for the 
control trajectory $\mathbf{Z}_{t}$ for various objectives specified on the trajectory $\mathbf{P}_{t}$. If the objective is only to influence the change in one period, the control variables must be set at

$$
\left\{\mathbf{Z}_{T}\right\}=\mathbf{H}^{-1}\left[\left\{\mathbf{P}_{T+1}\right\}-\mathbf{G}\left\{\mathbf{P}_{T}\right\}\right]
$$

If the objective is to have an effect at the end of time $t$ and $\left\{\mathbf{Z}_{t}\right\}$ is constant, we need to apply Equation (19) successively to find

$$
\left\{\mathbf{P}_{t}\right\}=\mathbf{G}^{t}\left\{\mathbf{P}_{0}\right\}+(\mathbf{I}-\mathbf{G})^{-1}\left(\mathbf{I}-\mathbf{G}^{t}\right) \mathbf{H}\{\mathbf{Z}\}
$$

If we have a target $\left\{\mathbf{P}_{t}\right\}$, this can be solved for $\left\{\mathbf{Z}_{t}\right\}$ to give (Willekens, 1978b, p. 49):

$$
\left\{\mathbf{Z}_{t}\right\}=\mathbf{H}_{t}^{-1}\left(\mathbf{I}-\mathbf{G}^{t}\right)^{-1}(\mathbf{I}-\mathbf{G})\left[\left\{\mathbf{P}_{t}\right\}-\mathbf{G}^{t}\left\{\mathbf{P}_{0}\right\}\right]
$$

In a sense $\mathbf{H}^{-1}$ serves to convert economic and other nondemographic variables into their demographic effects.

\section{CONCLUSION}

This chapter has passed in review generalizations of the life table applicable to a wide range of topics in sociology. If one tries to think through the probability that a man of 20 who is in the labor force will still be in the labor force 15 years later, one might start with a table showing labor force numbers at age 20 and age 35 . But even if the table has been made on the stationary model, the ratio of the number aged 35 to the number aged 20 means little-after all, many of those in the labor force at age 35 entered at an age older than 20. Expression (4), which applied here would be $l(35 / 20)=$ $l(35) l(20)^{-1}$, takes this and a variety of other transitions into account.

Manipulation of matrices by computer is as simple to program as manipulation of scalars, and this applies as much in the construction of the multidimensional life table in the first place as it does in the calculation of probabilities from the completed table.

The advent of these simple techniques presses us back one stage: to the collection of data, including information on events (such as deaths) and exposures (such as the resident population) in comparable form. The expanded data sources should include 
cross-classifications (for example, marital status by labor-force status by age) - both for events (marrying, joining the labor force, dropping out of it, and so on) and for the exposed population.

Although the methods described here draw somewhat more on mathematics than does the usual course on demographic techniques, they have the advantage of unifying a large part of the subject. One need not regard making an ordinary life table, a marriage table, a labor-force table, and an interregional migration table as separate problems. The technique here summarized deals with them as well as all other transitions. The main limit to their application is the Markov assumption that the history of the process affects each transition only through the state distribution immediately before that transition.

\section{REFERENCES}

CHIANG, C. L.

1960a "A stochastic study of the life table and its applications: I. Probability distributions of the biometric functions." Biometrics 16:618-635.

1960b "A stochastic study of the life table and its applications: II. Sample variance of the observed expectation of life and other biometric functions." Human Biology 32:221-238.

1961 "On the probability of death from specific causes in the presence of competing risks." Proceedings of the Fourth Berkeley Symposium on Mathematical Statistics and Probability 4:169-180.

1968 Introduction to Stochastic Processes in Biostatistics. New York: Wiley. CODDINGTON, E. A., AND LEVINSON, N.

1955 Theory of Ordinary Differential Equations. New York: McGraw-Hill. FEENEY, G. M.

1970 "Stable age by region distribution." Demography 7:341-348. FELLER, $\mathrm{W}$.

1941 "On the integral equation of renewal theory." Annals of Mathematical Statistics 12:243-267.

FIX, E., AND NEYMAN, J.

1951 "A simple stochastic model of recovery, relapse, death and loss of patients." Human Biology 23:205-241.

GANTMAGHER, F. R.

1959 The Theory of Matrices. K. Hirsch., Trans. New York: Chelsea. 
HOEM, J.

1975 "The construction of increment-decrement life tables: A comment on articles by R. Schoen and V. Nelson." Demography 12:661.

HOEM, J., AND FONG, M.

1976 A Markov Chain Model of Working Life Tables: A New Method for the Construction of Tables of Working Life. Working Paper No. 2. Copenhagen: Laboratory of Actuarial Mathematics, University of Copenhagen.

KITSUL, P.

1978 "On probabilistic approach to transition migration processes." Unpublished manuscript.

KRISHNAMOORTHY, S.

1978 "The demography of the life cycle." Unpublished doctoral dissertation, School of Public Health, Harvard University.

LE BRAS, H.

1971 "Equilibre et croissance de populations soumises à des migrations." Theoretical Population Biology 2:100-121.

MERTENS, $W$.

1965 "Methodological aspects of construction of nuptiality tables." Demography 3:317-348.

OECHSLI, F.

1971 "The parity and nuptiality problem in demography." Unpublished doctoral dissertation, University of California, Berkeley.

1975 "A population model based on a life table that includes marriage and parity." Theoretical Population Biology 7:229-245. ROGERS, A.

1975 Introduction to Multiregional Mathematical Demography. New York: Wiley.

ROGERS, A., AND LEDENT, J.

1976 "Increment-decrement life tables: A comment." Demography 13:287-290.

SCHOEN, R.

1975 "Constructing increment-decrement life tables." Demography 12:313-324.

SCHOEN, R., AND LAND, K. C.

1978 A General Algorithm for Estimating a Markov-Generated IncrementDecrement Life Table with Applications to Marital Status Patterns. Working Papers in Applied Social Statistics, WP7715, Urbana: University of Illinois. 
SCHOEN, R., AND NELSON, V.

1974 "Marriage, divorce, and mortality: A life table analysis." $D e-$ mography 11:267-290.

SINGER, B. AND SPILERMAN, S.

1976 "The representation of social processes by Markov models." American Journal of Sociology 82(1):1-54.

SMITH, D., AND KEYFITZ, N. (EDS.)

1977 Mathematical Demography: Selected Papers. Heidelberg: SpringerVerlag. SVERDRUP, E.

1965 "Estimates and test procedures in connection with stochastic models for deaths, recoveries and transfers between different states of health." Skandinavisk Aktuarietidskrift 40:184-211.

WILLEKENS, F.

1976a "Analytics of multiregional population distribution policy." Unpublished doctoral dissertation, Northwestern University.

1976b Optimal Migration Policies. Research memorandum RM-76-50. Laxenburg, Austria: International Institute for Applied Systems Analysis.

1977 "Sensitivity analysis in multiregional demographic models." Environment and Planning A(9):654-674.

1978a The Demography of Labor Force Participation. Research memorandum RM-78-17. Laxenburg, Austria: International Institute for Applied Systems Analysis.

1978b "Optimal control of dynamic population systems." Paper prepared for presentation at the Quetelet Chair Seminar, Catholic University of Louvain, Belgium.

WILLEKENS, F., AND ROGERS, A.

1976 Computer Programs for Spatial Demographic Analysis. Research memorandum RM-76-58. Laxenburg, Austria: International Institute for Applied Systems Analysis.

1977 More Computer Programs for Spatial Demographic Analysis. Research memorandum RM-77-30. Laxenburg, Austria: International Institute for Applied Systems Analysis. 


\section{THE AUTHOR}

Nathan Keyfitz, Andelot Professor of Sociology at Harvard University, is a member of the US National Academy of Sciences and the author of a number of books on mathematical demography, among them Introduction to the Mathematics of Population and Applied Mathematical Demography. 


\section{SELECTED PAPERS ON MIGRATION AND SETTLEMENT AT IIASA}

Andrei Rogers, editor, Migration and Settlement: Selected Essays, RR-78-6, reprinted from Environment and Planning A 10(5):469-617.

Andrei Rogers and Frans Willekens, Migration and Settlement: Measurement and Analysis, RR-78-13.

Frans Willekens and Andrei Rogers, Spatial Population Analysis: Methods and Computer Programs, RR-78-18.

Andrei Rogers, Migration Patterns and Population Redistribution, RR-80-7, reprinted from Regional Science and Urban Economics 9(1979):275-310.

Andrei Rogers, editor, Essays in Multistate Mathematical Demography, RR-80-10, reprinted from Environment and Planning A 12(5):485-622. 